\title{
Patience, patients, and the potential of medical preprints
}

\author{
Patrick S. Phelan \\ Washington University School of Medicine in St. Louis
}

May 14, 2019

Word Count (abstract): 133

Word Count (body): 4,331 


\begin{abstract}
Current debate over the merits of preprints for medical research turns on the potential benefits and harms to follow, often centered on purported differences that make clinical research unique among the sciences. Despite growing interest, existing discussions of this issue are generally brief and lack comprehensive appraisal of considerations and arguments. In this essay, I attempt a thorough analysis of the open questions for medicine regarding preprints, surveying existing concerns and claims, identifying what characteristics set medical research apart from its peers, and offering perspective on possible futures for medical preprints. I conclude that the extant claims of potential harms do not support recommendations of prohibition. Despite this, legitimate differences in the character and consequences of medical research give cause for more caution in the implementation of preprints than in other scientific fields.
\end{abstract}

\title{
Introduction
}

For the medical research community, one of the latest frontiers in the evolution of scientific "openness" is prepublication dissemination of research works, where "preprint" manuscripts take center stage. With increasing and lively discussion of this issue in the medical literature [1-12], writers have paid much attention to the longstanding use of preprints in other scientific fields and the existing interdisciplinary discussion of their potential advantages [13-19]. Generally accepted advantages of preprints include rapid, free, and open dissemination, establishment of priority, and connection to future works through citation [20]. The medical debate is nonetheless unresolved, held at various junctures by questions of how medicine and medical research differ from other applied sciences and disciplines, and how preprints might 
produce distinct consequences in distinct ways. A synthesis of considerations and analysis of arguments may help to clarify the current state of debate and frame the possible routes for resolution.

\section{Preliminaries and Premises}

Use of the term "preprint" in the recent medical literature comports with the definition given in the relevant Committee on Publication Ethics (COPE) Discussion Document: "a scholarly manuscript posted by the author(s) in an openly accessible platform, usually before or in parallel with the peer review process" [20]. Publishing database SHERPA/RoMEO distinguishes preprints in this sense from "postprints," which are peer-reviewed and revised (if necessary) manuscripts accepted for publication [21]. Although there has been some conflation of terms historically [22], this "pre-peer review" sense of "preprint" is the one I intend.

Throughout this paper I will refer to the relevant preprints as "medical preprints." By this I mean to include all research directly relevant to the practice of human medicine, including clinical research on living patients, human biological specimens, or patient medical records, as well as population level data gathered in epidemiology and public health research when relevant. Some "basic science" research (e.g. in neuroscience) may also meet this criterion, however my primary concern is with clinical research (i.e. that performed in the context of patient care or toward that immediate end). Some of this discussion may extend to related dissemination vehicles and open science practices (e.g. postprints, "open data"), but these will not be explicitly considered. 
For simplicity, I mean "benefits" and "harms" to refer to generally desirable and undesirable outcomes respectively. I expect that my choice of labels for consequences will be uncontroversial, so I will leave aside questions of perspective in judging desirability.

The discussion of medical preprints is largely one of future outcomes. Although a relatively small number of medical preprints already exist, the practice has not (yet) seen broad uptake and normalization within the field. As a result, most claims and their analyses are necessarily theoretical (though in some cases contesting the admissibility of external evidence from other fields' experiences). In such discussions, argumentative rigor must be maintained if any progress is to be made: claims and terms must be unambiguous, premises and assumptions must be made clear, and the validity of reasoning must be challenged and defended. I approach the present essay from this position, aiming to apply these principles to the discussion thus far and extract novel, meaningful conclusions.

More substantially, but beyond the scope of this discussion, there is an apparent dearth of predictive evidence relevant to the issue. Further empirical research, possibly both prospective and comparative across scientific fields, may prove essential to settling the questions discussed herein. Parallel to this aim, an ancillary goal of the present discussion is to ensure that such questions are sufficiently specified for a priori design of future studies.

\section{The Status Quo}

The current state of publishers' policies toward preprinting offers one perspective on the practice: among the most prominent general medical journals, PLoS Medicine has a permissive policy [9], The BMJ has a permissive-but-cautious policy [23], and The Lancet is currently 
continuing a trial period of permitting preprinting through a partnership with Elsevier's MedRN [7,24]; in contrast, both the New England Journal of Medicine and JAMA (and its network journals) retain permanent prohibitive or discouraging policies [25,26]. Publishers' policies across specific fields of clinical medicine are as yet uncharacterized in the literature, but notably some major publishers have permissive blanket policies (e.g. Elsevier [27], Taylor \& Francis [28]).

Despite increased discussion of the prospects for medical preprints in the literature, most such works are brief and, as I will argue, advance views without sufficient justification. The central claim of skeptics and opponents of medical preprints is roughly one of risk, asserting that they would be "dangerous" for patients, physicians, the public, the scientific community, or others, or that their pursuit reflects a lack of patience [4]. These writers often include claims against the potential benefits of medical preprints as well, but it seems fair to say these are secondary. As a good-faith attempt to summarize the common claim among opponents, consider the following thesis:

The Opponent's Thesis (OT): substantial uptake of preprinting in medical research poses significant risk of net harm, where component harms are specific to both the preprint medium and the medical-scientific field.

In so-naming the OT I do not mean to imply that all who voice concerns, skepticism, or advocate caution in uptake of preprinting necessarily oppose the practice outright. Rather, I take it that those who voice such concerns oppose the immediate wholesale uptake of preprinting, in 
the sense that medical research might embrace it to the same extent as other fields (e.g. physics, experimental psychology).

As justifications for the OT I offer the following: (1) only uptake that is substantial is of interest due to social significance of publishing practices (cf. the current debate) and heterogeneity of published work (in terms of applicability, significance, etc.); (2) it is net harm that must be implied, for the concerns that have been raised (and others conceivable) are not clearly so weighty as to render any countervailing benefits insufficient compensation; (3) the component harms must be specific to preprints, for otherwise they risk counting equally against other vehicles currently used for research dissemination; and (4) the component harms must be at least relatively specific to medical research in order to discount empirical evidence from the use of preprinting in other scientific fields.

The first two components of the OT appear prima facie reasonable, and the latter two have been expressed by opponents themselves [3,6]. In any case, the discussion herein will apply to similar formulations.

To evaluate the OT, we must first understand the meaningful ways in which preprinting might produce specific harms and in which medical research differs from other scientific fields. Only then can we properly appraise the risk of putative potential harms against the prospect of putative potential benefits.

\section{The Preprint Difference}

Understanding the situation of preprints within the dissemination sphere is crucial for assessment of specific consequences. The most obvious distinction is between preprints and 
published articles: published articles have been peer-reviewed and externally edited, whereas preprints have not and are often identical to submitted manuscripts prior to review. Opponents of preprints commonly cite this lack of peer review as a central motivator for concern, and it appears to be the primary justification for claims of specific harms. Although a basic and essential distinction, this fails to capture the full context provided by other forms of non-peerreviewed research dissemination: posters, text abstracts, and oral presentations at conferences. These vehicles are important for several reasons: first, they are (nearly) universally accepted and generally valued across diverse scientific fields; second, they generally undergo a less rigorous peer review process than traditional publication; third, their substance is not generally confined to the conference venue, as compendia of abstracts are commonly published in organizers' journals; fourth, their content is not generally confined to the conference venue, as media coverage is often allowed. We will return to some of these factors later.

One challenge to specificity is thus to clarify how preprints differ from these existing vehicles sufficiently to treat them as a medium sui generis. Several factors may enable opponents to meet this challenge. First, preprints need not be limited in length, and mature preprints (i.e. those ready for submission and formal peer review) typically contain more content and commentary than conference vehicles. Second, without preprint server curation, preprints lack even the minimal peer review accorded to conference vehicles. If conference-level peer review provides meaningful assurance of research quality, its absence in preprints may nonetheless be offset by the greater transparency in reporting, as mature preprints often include more detail in methodology and results; readers are then better equipped to appraise research quality for themselves. Some commentators have granted the comparison with non-publication vehicles, 
but objected that existing scientific attitudes toward them explicitly acknowledge their preliminary nature and temper interpretations appropriately, whereas these attitudes have not yet developed for preprints [5]. Granting that the claimed view toward non-publication vehicles probably does exist, there are two problems with this response: first, if lack of an adaptive cultural attitude is sufficient to block attempts at change, then much important progress may be forever forestalled; second, it is not clear that such an attitudinal correction can protect against all potential harms ascribed to preprints-in particular, the risk of psychological contamination and implicit bias [6] may still apply equally to non-publication vehicles. Opponents must demonstrate that such conclusions are sufficiently convincing to meet this challenge.

A second challenge to specificity concerns the more robust peer review that ostensibly distinguishes published works from their conference counterparts. When opponents leverage this formal peer review against preprints, a subtle but crucial conditionality is lost if we do not consider the proper counterfactual: In general, specific harms of preprints are conditional on shortcomings (e.g. errors, inappropriate interpretations) that are both present and would have been caught and corrected through peer review. These conditions bear on two external factors relevant to assessing the risks of preprinting: first the quality of research to be preprinted, and second the quality of traditional, formal peer review. Each of these factors is amenable to both empirical characterization and potential modification. As brief examples, the pool of works admissible to a given preprint server can be constrained by limiting submission to authors "endorsed" by peers or academic affiliation (as with arXiv [29]), and improvements in existing peer review processes can increase the proportion of errors corrected before publication. No doubt both of these conditions are met, and will continue to obtain, for a non-negligible 
proportion of published works. However, this does not entail that the same is true for the ensemble of potential preprints, nor would it be sufficient to produce meaningful harms.

\section{The Clinical Difference}

There is a common refrain among opponents of medical preprints that medicine, and more specifically medical scientific research, is different from other scientific fields in meaningful ways $[3,6]$. This claim is broad, intuitive, and almost undeniably true: whereas divisions between "applied" and "theoretical" pursuits exist in various fields (e.g. physics, math, statistics) the products of medical science are often directly and proximally aimed for application in individuals' lives through the sensitive (and sometimes intimate) context of patient care. However, this is not to say that medicine is unique, as applied disciplines such as engineering may produce implications for public benefit or harm that are similar in kind (notably, a dedicated engineering preprint server has been established in the form of engrXiv [30]).

There are at least two major ways the consequences of medical preprints might differ meaningfully from those in other fields: the first is impact, and the second is time-sensitivity.

Distinct impact follows directly from the fundamental difference: whereas the products of medical research yield effects on the health and welfare of patients, so too could medical preprints yield a net effect different from their traditionally published counterparts. However, this fundamental difference is in principle double-edged: if medical preprints can carry distinct risks of harm, so too can they carry distinct prospects of benefit. Merely identifying potential harms does not provide support for a course of action, as potential benefits must be identified for comparison. Importantly, preprint-specific mechanisms of impact would likely be limited to 
influences in individual behavior, as policy and organizational decisions would likely entail waiting for (or performing) formal peer review. Impacts on medical care would most clearly be effected at the level of physicians (and perhaps other clinicians) and patients. These will be explored later.

Time-sensitivity concerns the goal of preprints to avoid delay in dissemination of information and prevent delay-related harms (and we can generally consider these proportional in magnitude to the duration of delay). The significance of dissemination delay involved in traditional medical publication has been a common topic of debate $[10,12]$ and the subject of recent empirical study [31,32]. Ensuing harms might arise in two contexts: period-independent contexts, where publication delay produces harm unrelated to the relative time-situation of the delay, or period-dependent contexts, where delay produces harm directly related to the relative time-situation of the delay. Medically relevant period-independent contexts might include the treatment of conditions lacking short-term trends in incidence or prevalence (e.g. trauma), whereas period-dependent contexts might include responses to evolving public health phenomena (e.g. infectious disease outbreaks, pharmacovigilance). These types of timesensitivity are not wholly specific to medicine, as there are some clear examples of perioddependence in other fields (e.g. celestial phenomena in astronomy), but some mechanisms of period-dependence (such as pharmaceutical deployment) do appear specific to medicine, and the distinct impacts of medical research can be amplified by time-sensitivity. Interestingly, some opponents have asserted that significant delays in traditional publication of impactful medical research are either rare [10,12] or even potentially desirable [3], though both the duration and potential impacts of delay almost surely vary across medical subfields. 
The significance of time-sensitivity in medical research can be demonstrated through two examples. In a case study of preprint dissemination during active infectious disease outbreaks, Michael Johansson and colleagues describe the use and success of preprint communications during the Ebola and Zika virus epidemics of 2014-2017 [33]. During these events, preprinting enabled rapid communication of new data and analyses, despite only being used for a small proportion of traditionally published reports. For infectious diseases, the duration of outbreaks may be influenced by intentional clinical and public health measures for containment, adding another layer of complexity to period-dependence.

Another infectious disease context gave rise to a different sort of example in late 2009, when distribution of vaccines for $\mathrm{H}_{1} \mathrm{~N}_{1}$ influenza A began in anticipation of a "swine flu" pandemic. By late February 2010, Finnish physician Markku Partinen first recognized a possible association between a case of newly diagnosed narcolepsy and receipt of a particular adjuvanted $\mathrm{H}_{1} \mathrm{~N}_{1}$ vaccine formulation actively being distributed across Finland. Partinen and colleagues collected data on incident cases of narcolepsy nationally through the end of 2010, and assembled a compelling profile of narcolepsy as a complication of $\mathrm{H}_{1} \mathrm{~N}_{1}$ vaccination [34,35]. This work represents the starting point of a legacy spanning the last ten years, with details of the narcolepsy phenomenon and its management continuing to emerge in recent investigations [36]. Unfortunately, the team's reports would not appear in the peer-reviewed literature until their publication by PLOS ONE in March 2012, following lengthy delays while under consecutive review at the New England Journal of Medicine and The Lancet. In each case, the manuscripts underwent multiple rounds of review and revision before being rejected [37]. In retrospect, it is clear that the team could have disseminated their work as much as a full year faster through a 
preprint. The greater irony, however, is that the NEJM and Lancet policies at the time would have prohibited submission of preprinted work, so preprinting would have further spared the authors both review ordeals.

\section{Aims of Medical Research}

In clinical medicine, the primacy of patients' interests is a generally accepted point of professional integrity. Unsurprisingly, the question of potential benefit from preprints may be framed relative to this aim, asking "(how) will preprints benefit patients?" [10]. Acknowledging the lack of predictive evidence bearing on this question, I nevertheless find this construction unhelpful and unjustified. Benefits to patients are not clearly a necessary criterion for implementation of a practice, even if we maintain primary consideration of their interests. Benefits represent only one face of consequences, and a lack of harm to patients is a logically sufficient criterion to safeguard their interests. This is instructive when we consider that there are likely other parties for whom benefits and harms are relevant: researchers (as both authors and readers), the general public, physicians and other clinicians all have potentially relevant interests. For authors, perhaps the most general are the ability to establish priority and demonstrate interval progress in one's work [19]. Contrary to what some opponents imply [4], these interests are not irrelevant merely because they are subordinate to patients'. There may also be "intangible" consequences worth considering, such as pursuit of scientific virtue in attempting transparent and open dissemination of research works.

An alternative framing is one of correction, asking "what problem are preprints intended to solve?" [6]. Rather than straining credulity to imply a flawless status quo, this construction is 
probably best interpreted as discounting the plausibility of putative potential benefits. This appears implicitly similar to asserting a lack of need to adopt preprinting, but still must face the response that specific tangible benefit (or fulfillment of need) is not logically necessary for justification. Whatever its intended interpretation, this framing has historically been posed in opposition to preprinting in other scientific fields prior to its adoption [38].

\section{Plausibility of Putative Specific Harms}

Opponents have proposed various putative harms (or more accurately independent means of harm) to potentially arise from uptake of medical preprinting. These include harms to patients mediated through the actions of physicians, patients, and "the press" (popular news media), and through compromise of public trust in science. I should also note that some opponents' arguments appear to suffer from general problems such as contradictory premises (e.g. that physicians will not substantially attend to preprinted works, yet will do so sufficiently to influence their practice patterns). Additional putative harms or means have been presented as well, but their non-specificity to medical research is trivially demonstrable (e.g. preprint citation will derange reference schemes, preprint posting will be susceptible to abuse by "less scrupulous researchers" and lead to unspecified harms $[6,12])$. Rather than addressing these sorts of concerns, I instead focus on the medicine-specific considerations below.

In evaluating each of these through the lens of the OT we should bear in mind several things: the specificity criteria, the necessarily conditionality for attributable impact, and most importantly the double-edged nature of impact-each of these has the potential to be a means of benefit rather than harm, and predictions of one over the other must be justified. 
1. Physician-mediated harm: The prediction that physicians might err or be misled into harming their patients is quite grim, but this is a central claim directed in opposition to preprints. Proposed mechanisms for this route to harm include physicians' overrating of study importance, their failure to detect objective errors in reporting, and their psychological contamination by mere exposure to preprinted material. Here we can illustrate the conditionality necessary for specificity: where a physician would have also overrated the peer-reviewed version of a preprinted study, or where the same error(s) would persist undetectable through peer review, these outcomes cannot be specifically attributed to preprinting. Although peer review can encourage conservatism in authors' claims and identify errors to be corrected before publication [12], these functions must alter manuscripts sufficiently to change outcomes in order to support these mechanisms-otherwise, if physicians also overrate results and overlook errors in published works (as assuredly happens), the processes are consequentially equivalent. Granting even minimal confidence in physicians should imply that they have individual professional standards for what new research results are sufficient to change their behavior-that is, what counts as "practice-changing." Generally, such works should provide definitive, well-supported conclusions [39] and inspire confidence in their implementation. Assuming physicians hold similar conceptions, this seems a high bar for research reports, and likely only a small minority of all contemporary medical research publications would meet it (even on an imperfect appraisal). The alternative that physicians' standards for "practice-changing" reports are much lower is troubling, but 
would yield the sort of vacillation in individual practice patterns that could easily harm patients. Regardless, claims that physician decision-making is sufficiently susceptible to this sort of misclassification error should be supported by empirical behavioral research.

2. Patient-mediated harm: It is also possible that patients might harm themselves by modifying their medical care or employing their own interventions. Patients can discontinue or alter their own adherence to outpatient medication or other therapy, and can initiate self-treatment with behavioral and dietary changes, non-pharmaceutical substances (such as dietary supplements), and some drugs (most obviously those legally available non-prescription). A different concern is that patients may "demand" therapies based on preprinted information, and physicians may feel obligated to at least address the issue and perhaps pressured to comply without familiarity or confidence in the evidence. Here there are intrinsic potential harms associated with clinical encounters, such as lost time or rapport, as well as connection to physician-mediated harm if unjustified patient demands are met. Patient actions outside of clinical encounters may be the more plausible means for harm, but reasons for skepticism remain to be addressed, including why preprints should exert greater or significant additional influence on patients compared to the many sources of incorrect and misleading health information already available.

3. Press-mediated harm: Opponents claim that medical research is of greater public interest and intellectual accessibility than that in fields such as physics, and for the sake 
of argument let us assume this is both true and reflected in the volume of press coverage between scientific fields. It seems hardly contestable that the press frequently distorts the messages of formally published research, whether inadvertently or intentionally, in ways potentially harmful to patients (and physicians). Importantly, this applies to both works that have passed formal peer review and those that have not, such as conference presentations. Recalling the challenge to specificity that non-publication vehicles present for preprint opponents, this means for harm is particularly interesting. Given that the press is liable to distort even peer-reviewed works, it seems undeniable that they might also distort preprinted reports. However, by negative argument one may ask why these works should not then be restricted from the media even after formal peer review-for example, by conferences' total embargo of materials distribution, medical researchers' refusal of interviews, and scholarly advocacy for journalistic self-regulation of scope. This last suggestion bears on two further important points: first, harms attributable to the press are a concern for the press, not only for researchers, and second, there is a widelyheld belief that journalistic freedom has social value. These observations imply that solutions to press-mediated harms require collaboration between researchers and journalists, and that cloistering the scientific community is a questionable response. Opponents might argue that preprints nonetheless present the possibility of marginal (additional) harm if adopted; this claim is plausible, but applies to all future research works. Adequate specificity to preprints requires grounds to expect press-mediated harms to be greater for preprints than for existing vehicles. If peer review alone is taken to ground this expectation, then opponents must justify the current practice of non- 
publication dissemination at conferences, especially because press coverage of these works may not explicitly note the lack of formal peer review.

4. Erosion of public trust: Catherine Otto suggests that medical preprints have the potential to "destroy public confidence in medical research" [10]. A plausible reading takes her to mean an unjustified loss of trust, wherein the public develops a distorted image of the research process or its rigor through exposure to preprints. This would plainly count as a social harm in itself, and may have special significance for medical research because it presents a risk for further patient-mediated harms if the public disengages from beneficial medical practices. However, it is unclear why we should expect access to preprints to be more influential toward this end than the various scandals of scientific misconduct, publication retraction, secrecy [40], and position reversal $[41,42]$ that already occur. Opponents might deny that preprints are any more troublesome than existing vehicles, but maintain that the marginal (additional) risk they present should be avoided. However, even if one assigns the status quo to a privileged position of reference and claims that any additional harms cannot be justified—regardless of additional benefits—-this cannot plausibly hold for merely potential harms; such a position only serves to arbitrarily entrench any current system against change. On another interpretation, a justified loss of trust might occur where adoption of medical preprints makes clear any shortcomings or failures of peer review-assessing this outcome is more complex, as its intentional avoidance amounts to public deception, and its acceptance could nonetheless manifest other means of harm. 


\section{Conclusions}

Defenders of the Opponent's Thesis face a challenging argumentative task. They must demonstrate that the preprint medium should be considered differently from existing research dissemination vehicles (peer-reviewed or otherwise), that manifestation of any given means of harm is plausible, that the outcomes of those means are likely to be harms rather than benefits, that the sum of likely outcomes is a net harm of significant magnitude, and that the likely means and harms are sufficiently specific to medical research to discount evidence to the contrary from other sciences. Arguments attempting this challenge must be thorough and coherent, and those presented in the medical literature thus far have failed.

Furthermore, any successful defense of the OT should be interpreted in a specific context for adopting preprinting, effectively seeking to determine whether the expected net harms can be feasibly mitigated. Analysis of what this task might entail is left for future study.

Although we cannot justifiably accept the OT, there are clear and compelling reasons to consider medical research distinct in nature from the sciences best known for preprinting, and prudence requires that we think carefully and communicate clearly to guide progress with medical preprints. 


\section{References}

1. Lauer, M.S.; Krumholz, H.M.; Topol, E.J. Time for a prepublication culture in clinical research? The Lancet 2015, 386, 2447-2449.

2. Tabor, E. Prepublication culture in clinical research. The Lancet 2016, 387, 750.

3. Fuster, V. Pre-Publication in Medical Research. J. Am. Coll. Cardiol. 2016, 67, 883-884.

4. Fuster, V. Who Truly Benefits From Pre-Publishing and Rushing Publishing? J. Am. Coll. Cardiol. 2017, 70, 3199-3200.

5. Nallamothu, B.K.; Hill, J.A. Preprints and Cardiovascular Science. Circulation 2017, 136, 1177-1179.

6. Maslove, D.M. Medical Preprints-A Debate Worth Having. JAMA 2018, 319, 443.

7. Kleinert, S.; Horton, R. Preprints with The Lancet : joining online research discussion platforms. The Lancet 2018, 391, 2482-2483.

8. Sheldon, T. The impact of preprint on media reporting of science. The Lancet 2018, 392, 1194.

9. Peiperl, L.; the PLOS Medicine Editors Preprints in medical research: Progress and principles. PLOS Med. 2018, 15, e1002563.

10. Krumholz, H.M.; Ross, J.S.; Otto, C.M. Will research preprints improve healthcare for patients? BMJ 2018, k3628.

11. Oakden-Rayner, L.; Beam, A.L.; Palmer, L.J. Medical journals should embrace preprints to address the reproducibility crisis. Int. J. Epidemiol. 2018, 47, 1363-1365.

12. Leopold, S.S.; Haddad, F.S.; Sandell, L.J.; Swiontkowski, M. Clinical Orthopaedics and Related Research, The Bone \& Joint Journal, the Journal of Orthopaedic Research, and The Journal of Bone and Joint Surgery Will Not Accept Clinical Research Manuscripts Previously Posted to Preprint Servers*: J. Bone Jt. Surg. 2019, 101, 1-4.

13. Tracz, V.; Lawrence, R. Towards an open science publishing platform. FioooResearch 2016, 5, 130.

14. Chalmers, I.; Glasziou, P. Should there be greater use of preprint servers for publishing reports of biomedical science? FioooResearch 2016, 5, 272.

15. Powell, K. The Waiting Game. Nature 2016, 530, 148-151.

16. Kaiser, J. The preprint dilemma. Science 2017, 357, 1344-1349.

17. Bourne, P.E.; Polka, J.K.; Vale, R.D.; Kiley, R. Ten simple rules to consider regarding preprint submission. PLOS Comput. Biol. 2017, 13, e1005473.

18. Heathers, J. Why I Love Pre-Prints. James Heathers 2018.

19. Sarabipour, S.; Debat, H.J.; Emmott, E.; Burgess, S.J.; Schwessinger, B.; Hensel, Z. On the value of preprints: An early career researcher perspective. PLOS Biol. 2019, 17, e3000151.

20. Preprints | Committee on Publication Ethics: COPE Available online: https://publicationethics.org/resources/discussion-documents/preprints (accessed on Apr 30, 2019).

21. SHERPA RoMEO Colours, Pre-print, Post-print, Definitions and Terms Available online: http://www.sherpa.ac.uk/romeoinfo.html (accessed on Apr 25, 2019).

22. Tomaiuolo, N.G.; Packer, J.G. Searcher. October 2000,.

23. Publishing model | The BMJ Available online: https://www.bmj.com/aboutbmj/publishing-model (accessed on Apr 30, 2019). 
24. Preprints with The Lancet Available online:

https://papers.ssrn.com/sol3/JELJOUR_Results.cfm?form_name=journalBrowse\&journal_id=318 4962 (accessed on Apr 30, 2019).

25. Editorial Policies | About NEJM Available online: https://www.nejm.org/aboutnejm/editorial-policies (accessed on Apr 30, 2019).

26. Instructions for Authors Available online:

https://jamanetwork.com/journals/jama/pages/instructions-for-authors (accessed on Apr 30, 2019).

27. Article Sharing Available online: https://www.elsevier.com/about/policies/sharing (accessed on Apr 30, 2019).

28. How to share your article \& use different versions on different platforms Available online: https://authorservices.taylorandfrancis.com/sharing-your-work/ (accessed on Apr 30, 2019).

29. The arXiv endorsement system | arXiv e-print repository Available online:

https://arxiv.org/help/endorsement (accessed on Apr 30, 2019).

30. engrXiv Preprints Available online: https://engrxiv.org/ (accessed on Apr 30, 2019).

31. Bauchner, H. The Rush to Publication: An Editorial and Scientific Mistake. JAMA 2017, 318, 1109-1110.

32. Qunaj, L.; Jain, R.H.; Atoria, C.L.; Gennarelli, R.L.; Miller, J.E.; Bach, P.B. Delays in the Publication of Important Clinical Trial Findings in Oncology. JAMA Oncol. 2018, 4, e180264.

33. Johansson, M.A.; Reich, N.G.; Meyers, L.A.; Lipsitch, M. Preprints: An underutilized mechanism to accelerate outbreak science. PLOS Med. 2018, 15, e1002549.

34. Partinen, M.; Saarenpää-Heikkilä, O.; Ilveskoski, I.; Hublin, C.; Linna, M.; Olsén, P.; Nokelainen, P.; Alén, R.; Wallden, T.; Espo, M.; et al. Increased Incidence and Clinical Picture of Childhood Narcolepsy following the $2009 \mathrm{H}_{1} N_{1}$ Pandemic Vaccination Campaign in Finland.

PLOS ONE 2012, 7, e33723.

35. Nohynek, H.; Jokinen, J.; Partinen, M.; Vaarala, O.; Kirjavainen, T.; Sundman, J.;

Himanen, S.-L.; Hublin, C.; Julkunen, I.; Olsén, P.; et al. ASoz Adjuvanted AHıN1 Vaccine Associated with an Abrupt Increase in the Incidence of Childhood Narcolepsy in Finland. PLoS ONE 2012, 7, e33536.

36. Doshi, P. Pandemrix vaccine: why was the public not told of early warning signs? BMJ 2018, k3948.

37. Kelland, K. Special Report: How vaccine scares cast shadows over science. Reuters 2013.

38. Nguyen, T.M. Chemical \& Engineering News. January 19, 2019,.

39. Brassey, J. Finding practice-changing research evidence Available online:

https://blogs.bmj.com/bmjebmspotlight/2018/12/o3/how-do-you-find-practice-changingresearch-evidence/ (accessed on Apr 21, 2019).

40. Marquardsen, M.; Ogden, M.; Gøtzsche, P.C. Redactions in protocols for drug trials: what industry sponsors concealed. J. R. Soc. Med. 2018, 111, 136-141.

41. Skolnik, N. No Wonder No One Trusts Us. JAMA Intern. Med. 2017, 177, 1253.

42. Prasad, V.; Cifu, A. Medical Reversal: Why We Must Raise the Bar Before Adopting New Technologies. Yale J. Biol. Med. 2011, 84, 471-478. 